\title{
Observer variability in histopathological reporting of malignant bronchial biopsy specimens
}

\author{
R A Burnett, J Swanson Beck, S R Howatson, F D Lee, A M Lessells, K M McLaren, \\ $S$ Ogston, A J Robertson, J G Simpson, G D Smith, H B Tavadia, F Walker
}

\begin{abstract}
Aims-To evaluate the ability of histopathologists to classify lung carcinomas on bronchial biopsy material using the current World Health Organisation (WHO) classification.

Methods-Eleven histopathologists each reviewed 100 randomly selected bronchial biopsy specimens which had originally been reported as showing lung carcinoma. A single haematoxylin and eosin stained section from each case was circulated and a standard proforma completed. These were analysed using $\kappa$ statistics.

Results-The histopathologists were excellent at distinguishing between small cell and non-small-cell carcinoma $\kappa=0 \cdot 86$ ), but not so good at subclassifying the non-small cell carcinoma group $\kappa=0 \cdot 25)$.

Conclusions-The clinically important distinction between small cell and nonsmall cell carcinoma of the lung is reliably made by competent histopathologists even on limited material.
\end{abstract}

(F Clin Pathol 1994;47:711-713)

It falls to the histopathologist or cytopathologist to provide the "tissue diagnosis" of bronchial or lung carcinoma. Most pathologists use a classification based on the World Health Organisation's (WHO) second classification $(1981)^{1}$ (table 1). The subsequent treatment of lung cancer by surgery or radiotherapy is determined largely by clinical and pathological staging-for example, mediastinoscopy and biopsy-but it is most important to separate the small cell undifferentiated carcinoma group (classic oat cell carcinoma and the larger "intermediate cell" type) which is regarded as neuroendocrine in origin from all other types of lung cancer (non-small cell carcinoma) as the treatment and prognosis are completely different; most oncology centres use specific chemotherapy for small cell carcinoma in place of surgery.

The tissue diagnosis of lung cancer is usually made on material obtained by bronchial biopsy, or by the cytological examination of sputum, or bronchial washings or brushings. In this study we decided to review bronchial biopsy material in an attempt to evaluate the degree of confidence which can be given to the histological classification of lung cancer on such material by non-specialist histopathologists and thereby determine the "robustness" of the existing terminology and classification.
Table 1 Histological classification of lung tumours (WHO, 1981)

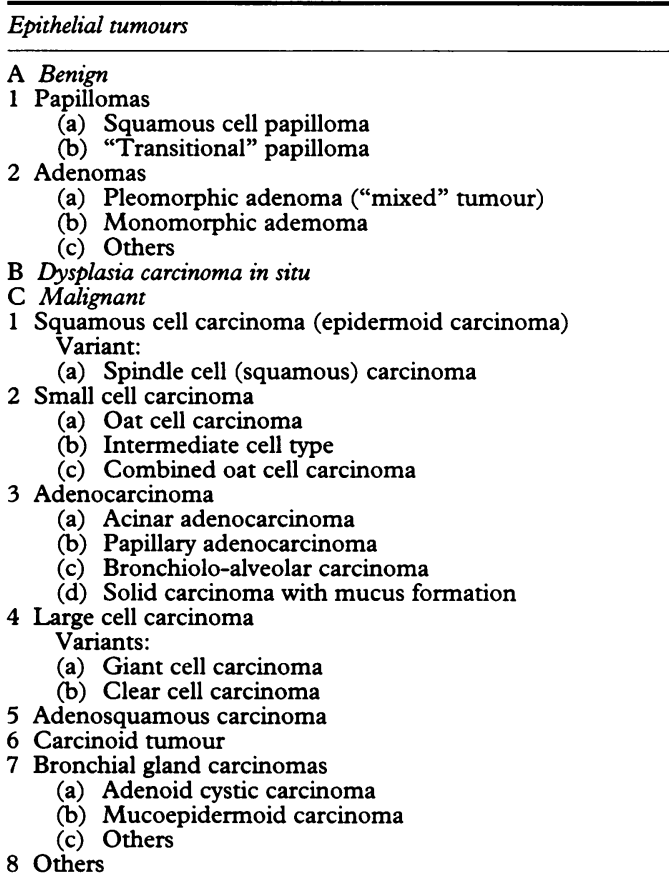

The WHO classification ${ }^{1}$ is based on tumour differentiation as seen in routinely produced sections examined by light microscopy and it does not take into account any additional evidence of differentiation which may become apparent using other more specialised techniques, such as immunohistochemistry or electron microscopy. It has also long been recognised that lung cancers are notoriously heterogeneous in their differentiation, different areas of the same tumour expressing completely different phenotypes. ${ }^{2}{ }^{3}$ In biopsy work only a tiny amount of tumour is present and, in practice, more than one differentiation pattern is rarely seen. We therefore decided that the circulation of a single haematoxylin and eosin stained section from each case would give a reasonable indication of our ability to assess tumour differentiation.

\section{Methods}

Eleven histopathologists took part in this study. As in our previous studies ${ }^{45}$ they had been chosen to represent Scottish histopathology as a whole. Only one (RAB) had any special expertise in respiratory pathology. The panel members came from pathology laboratories in Aberdeen $(n=2)$, Dundee $(n=2)$, Edinburgh $(n=2)$, Airdrie $(n=1)$, Stirling $(n=1)$ and Glasgow $(n=3)$. All of the participants were of consultant grade, but they varied in years of experience (nine to 29 years) and the nature of their substantive post 
Slide no:

(Please put tick in appropriate boxes)

Small cell carcinoma

Non small cell carcinoma

If non small cell carcinoma is it:

squamous

adenocarcinoma

large cell undifferentiated

Other State type

Re: Diagnosis

Very high level of confidence $=1$

Some doubt as to precise category $=2$

Pathologist code:

Figure Bronchial biopsy pathology

(university staff $\mathrm{n}=4$, NHS staff $\mathrm{n}=7$ ).

Ten of the 11 participants were asked to extract 10 consecutive cancer positive bronchial biopsy specimens from the files of their respective laboratories, starting from an arbitrary date of their own choosing. The 100 haematoxylin and eosin stained sections thus obtained were then randomly allocated and renumbered. The sections were circulated, in batches, round the 11 histopathologists who then completed a simple proforma (figure) for each section examined. The completed proformas were centrally collated.

The main problem with the analysis of studies of this kind is the lack of knowledge of the "correct" diagnosis for each slide. In some studies an "expert peer group" diagnosis has been used. In this study, as in some of our previous work, ${ }^{45} \kappa$ statistics were used to analyse the results. Kappa statistics are a measure of overall agreement and do not require any assumption concerning the "correct" diagnosis. They include a correction for the amount of agreement which would be expected by chance alone. The value of $\kappa$ can range from $-1 \cdot 0$ to $+1 \cdot 0$. A value of 0 indicates chance agreement only, while a value of 1.0 would indicate perfect agreement. A negative $\kappa$ value would indicate systematic disagreement between observers. It is generally accepted that a $\kappa$ value of 0.75 or higher reflects excellent agreement, $\kappa$ of 0.4 to 0.75 fair to good agreement, and $\kappa$ values of less than 0.4 poor agreement.

The participants were allowed to grade their responses into two degrees of confidence. Grade I indicated a very high degree of confidence, whereas grade II indicated doubt as to the precise classification of the tumour. The reason for this decision was the nature of the biopsy material itself. In some of the biopsy specimens the amount of tumour tissue present was so small that accurate classification was risky if not impossible. In others (particularly small cell tumours) the degree of traumatic artefact made the distinction between carcinoma, lymphoma, or even chronic inflammatory infiltration very difficult. In addition, while the original WHO lung cancer classification $(1967)^{6}$ placed solid large cell carcinomas which produced mucin into the large cell undifferentiated category, the more recent second WHO lung cancer classification (1981) moved this group of tumours out of the large cell undifferentiated group and into the adenocarcinoma group. Thus in the absence of a stain for epithelial mucin to examine, the diagnosis of large cell anaplastic carcinoma could only be made confidently up to grade II.

\section{Results}

The results of the study are shown in tables 2 and 3. The classification of carcinomas as small cell type was made with a very high degree of accuracy (overall $\kappa=0 \cdot 86$ ). In those cases where the biopsy specimen was adequate and little traumatised and the confidence level was grade $I$, the $[\kappa]$ value reached 0.97 -almost perfect agreement. Even where the confidence level was given as grade II, the $\kappa$ value achieved was $0 \cdot 70$.

Because of the problems already alluded to, the subclassification of the non-small cell tumours was less satisfactory. Adenocarcinoma was the differentiation pattern best recognised with a $\kappa$ value of $0 \cdot 58$. However, a point of real difficulty was the inability of the observers to distinguish between very poorly differentiated adenocarcinoma and large cell undifferentiated carcinoma without the aid of mucin stains.

The WHO criteria for the diagnosis of squamous cell carcinoma are strict, requiring either keratin production or the presence of intercellular bridges ("prickles"). It is clear from the poor $\kappa$ results that some observers were applying these criteria much more strictly than others.

The large cell undifferentiated group was reported least consistently in this study ( $\kappa=0.22$ for grade I competence and 0.17 for both competence groups together).

Finally, in all 100 cases a diagnosis of carcinoma of one type or another was sustained, suggesting that false positive diagnosis of lung cancer on bronchial biopsy material is not a problem in Scottish histopathology laboratories at the present time.

Table 2 Small cell undifferentiated carcinoma compared with non-small cell carcinoma

\begin{tabular}{ll}
\hline & $\kappa$ value \\
\hline All sections & 0.86 \\
Grade I confidence & 0.97 \\
Grade II confidence & 0.70 \\
\hline
\end{tabular}

Table 3 Subcategorisation of non-small cell carcinoma

\begin{tabular}{lllll}
\hline & & \multicolumn{3}{c}{$\begin{array}{l}\text { Large cell } \\
\text { Adeno- }\end{array}$} \\
& Squamous & $\begin{array}{l}\text { Andfer- } \\
\text { carcinomatiated }\end{array}$ & Overall \\
\hline All sections & 0.30 & 0.28 & 0.17 & 0.25 \\
Grade I confidence & 0.37 & 0.58 & 0.22 & 0.36 \\
\hline
\end{tabular}




\section{Discussion}

In the United Kingdom lung carcinoma kills more people than carcinoma of any other organ. Bronchial biopsy is an efficient way for the clinician to achieve a tissue diagnosis. Apart from providing a firm diagnosis, the pathologist will be asked to indicate whether the tumour is of small cell or non-small cell type because, with a small number of exceptions, such as well differentiated bronchial gland carcinomas, the type and degree of differentiation of non-small cell carcinoma does not affect subsequent staging, management, and treatment, although there may be some long term prognostic implications-well differentiated squamous carcinoma, for example, tends to metastasise late and carries a better prognosis than adenocarcinoma or large cell undifferentiated tumours. Small cell undifferentiated carcinomas, however, are usually treated completely differently, chemotherapy being offered instead of surgery, irrespective of the clinical stage.

We have shown that histopathologists are extremely consistent and reliable in their ability to distinguish between small cell carcinoma and other lung cancers, even in sometimes very limited and often badly traumatised biopsy material. They are, however, much less consistent in their ability to subclassify the large cell tumours into squamous carcinoma, adenocarcinoma, and undifferentiated categories. This is because large cell undifferentiated carcinoma of the lung is not regarded as a specific entity, but represents squamous carcinomas and adenocarcinomas whose poor degree of differentiation precludes their inclusion into a more specific diagnostic category, ${ }^{7}$ especially when very limited material is available for study. It is therefore not too surprising that the $\kappa$ values obtained by the group were very much lower when they attempted to subclassify the non-small cell group.

The 100 tumour positive cases used in this study were "pulled" at random from the histology files of several different laboratories. The presence of carcinoma was confirmed in all of them. This suggests that false positive reporting of lung carcinoma on bronchial biopsy material is not a major problem for histopathologists.

The Scottish Panel for the Consistency of Histopathological Reporting was set up by Professor J Swanson Beck in 1987. This study was funded by the Scottish Office Home and Health Department (CRAG) and Tayside Health Board. We are grateful to Mrs Joyce Duncan and Miss Margaret Brough for administrative and secretarial assistance.

1 World Health Organisation. Histological typing of lung tumours. International histological classification of tumours. No 1. 2nd edn. Geneva: WHO, 1981 .

2 Willis RA. Epithelial tumours of the trachea, bronchi and lung. In: Pathology of tumours. 3rd edn. London Butterworths, 1960:369.

3 Roggli VL, Vollmer RT, Greenberg SD, McGavron MH, Spjut HJ, Yesner R. Lung cancer heterogeneity: A blinded randomized study of 100 consecutive cases. Hum Pathol 1985;16:569-78.

4 Robertson AJ, Anderson JM, Beck JS, Burnett RA Robertson AJ, Anderson JM, Beck JS, Burnett RA,
Howatson SR, Lee FD, et al. Observer variability in histopathological reporting of cervical biopsy specimens. f Clin Pathol 1989;42:231-8.

5 Robertson AJ, Beck JS, Burnett RA, Howatson SR, Lee FD, Lessells AM, et al. Observer variability in histological reporting of transitional cell carcinoma and epithelia dysplasia in bladders. $\mathcal{f}$ Clin Pathol 1990;43:17-21.

6 World Health Organisation. Histological typing of lung tumours. 1st ed. International histological classification of tumours. No 1. Geneva: WHO, 1967.

7 Chuang MT, Marchevsky A, Teirstein A, Kirschner PA, Kleinerman J. The diagnosis of lung cancer by fibreoptic bronchoscopy. 1. Problems in the histologic classification of non-small cell carcinomas. Thorax 1984;39. $175-8$. 